\title{
NEFRECTOMÍA PARCIAL A CIELO ABIERTO Y LAPAROSCÓPICA: NUESTRA EXPERIENCIA EN 254 CIRUGÍAS
}

\author{
José J. Rozanec 1,2, Carlos Ameri ${ }^{3}$, Pablo Holst², Marcelo Featherston', Cayetano Vallone', Pablo \\ Atchabahián², Alberto Hernández', Alejandro Nolazco',2, Jorge Ares' y Osvaldo Mazza3.
}

'Hospital Británico de Buenos Aires. Argentina.

${ }^{2}$ Hospital Universitario Austral, Buenos Aires. Argentina.

${ }^{3}$ Hospital Alemán. Buenos Aires. Argentina.

Resumen.- OBJETIVOS: La mayoría de los tumores de riñón se detectan actualmente como una masa renal pequeña en pacientes asintomáticos, por el uso generalizado de la ecografía y otras modalidades no invasivas de exploración abdominal. La cirugía conservadora de riñón de estas masas renales pequeñas es el tratamiento de elección, tanto en pacientes con el riñón contralateral sano como en aquellos pacientes que presentan algún grado de deterioro en la función renal o son monorrenos funcionales o anatómicos. Revisamos retrospectivamente nuestra experiencia en cirugía conservadora de riñón en masas tumorales, a cielo abierto y por la vía laparoscópica.

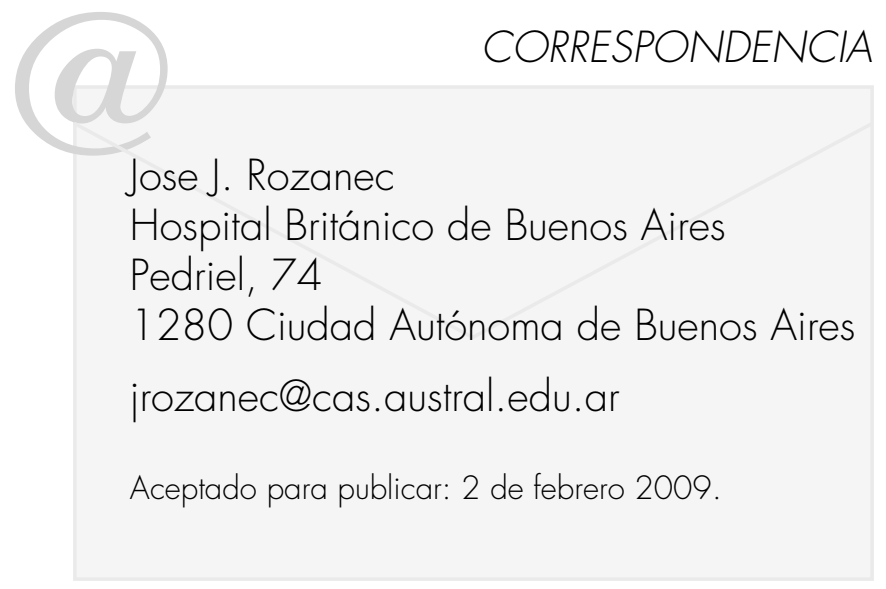

MÉTODO: Se revisaron las historias clínicas de aquellos pacientes a los cuales se diagnosticó una masa ocupante renal y se los trató con cirugía conservadora renal en el Hospital Británico de Buenos Aires y Hospital Alemán desde el año 1995 y el Hospital Universitario Austral desde el año 2000. Se tabularon todos los datos de interés de 246 pacientes, se analizaron las técnicas empleadas, complicaciones y resultados y se compararon con otras series de referencia.

RESULTADOS: Se realizaron 254 cirugías conservadoras de riñón. La cirugía a cielo abierto fue realizada en 220 casos y la nefrectomía parcial laparoscópica desde el año 2001 en 34 pacientes. La indicación fue electiva o relativa en 236 pacientes, 8 pacientes presentaron tumores bilaterales y hubo 18 cirugías en pacientes monorrenos. El clampeo del pedículo se realizó en 168 procedimientos, con hibernación en 43 pacientes con un tiempo promedio de 24,7 minutos. El promedio del tamaño tumoral tratado fue de $3,49 \mathrm{~cm}$. El examen histopatológico demostró un carcinoma renal en 193 procedimientos, siendo el porcentaje de lesiones benignas del 24\% (n=61). Un paciente presentó márgenes quirúrgicos positivos en diferido, se le realizó la nefrectomía al mes y no se constató tumor residual. Un paciente presentó una imagen pseudotumoral del lecho quirúrgico por TAC y RMN, se realizó una nefrectomía sin hallazgo de tumor residual. Se presentaron complicaciones postoperatorias en 20 pacientes 17,9 $\%)$. El control oncológico en el $84 \%$ de los pacientes con un promedio de 46,6 meses demostró una progresión con metástasis en 8 pacientes. La recurrencia local se observó en 4 pacientes (2\%). Un paciente desarrolló un tumor en el riñón contralateral a los 20 meses y otro a los 10 años. La sobrevida específica por cáncer fue del $98 \%$ y la sobrevida global del 95\%. En el último año el abordaje laparoscópico duplicó las indicaciones de todos los años previos. 
CONCLUSIONES: La cirugía conservadora renal, actualmente considerada el gold standard del tratamiento de los tumores renales pequeños, es una técnica segura y confiable para el tratamiento de dichos tumores. La evolución tecnológica y el entrenamiento laparoscópico nos permite para casos seleccionados reproducir la cirugía a cielo abierto por la vía laparoscópica, obteniendo similares resultados.

Palabras clave: Nefrectomía parcial. Tumores renales. Laparoscopía.

Summary.- OBJECTIVES: The majority of renal cell carcinomas are now incidentally detected as small renal masses in asymptomatic patients due to the widespread use of ultrasound and new improved noninvasive abdominal imaging modalities. Nephron-sparing surgery is the treatment of choice for patients with small renal masses in presence of normal contralateral kidney or in presence of an anatomic or functional solitary kidney. We reviewed retrospectively our experience in open and laparoscopic partial nephrectomy.

METHODS: The records for all patients who underwent nephron-sparing surgery for a renal mass since 1995 at British Hospital of Buenos Aires and Hospital Aleman and since 2000 at Hospital Universitario Austral were reviewed. The most relevant data of 246 patients were collected, with special focus on demographic data, operative details, pathology results, complications and outcome in cancer control.

RESULTS: We have performed 254 nephron-sparing surgeries. Open partial nephrectomy was performed in 220 procedures and the laparoscopic partial nephrectomy since 2001 in 34 patients. The indication was elective or relative in 236 patients with 8 patients with bilateral tumors and 18 tumors in a solitary kidney. The pedicle was clamped in 168 procedures with hypothermia in 43 patients. Mean ischemia time was 24.7 minutes. Average tumor size was $3.49 \mathrm{~cm}$. The pathologic findings demonstrate a carcinoma in 193 cases and benign lesions in 61 patients (24\%). One patient presented a positive surgical margin in the pathologic examination, but subsequent nephrectomy was negative for residual tumor. One patient presented a pseudo-tumoral mass on follow-up on CT scan and MRI, but nephrectomy was negative for residual tumor. There were postoperative complications in 20 patients (7.9\%). Oncologic follow up was available in $84 \%$ of the patients for an average of 46.6 months showing progression with metastasis in 8 patients. Local recurrence was observed in 4 patients (2\%). One patient developed a tumor in the contralateral kidney 20 months after partial nephrectomy and another one 10 years later. The cancer specific survival and overall survival rates were $98 \%$ and $95 \%$ respectively. In the last year the laparoscopic approach duplicated the indications of all previous years.

CONCLUSIONS: Open partial nephrectomy is considered nowadays the gold standard treatment of small renal masses, and in our experience it is a safe and effective technique of treatment of these tumors. The evolution of the technology and the acquirement of laparoscopic skills allow us in selected cases to duplicate the open approach in a laparoscopic way, obtaining similar results.

Keywords: Partial nephrectomy. Kidney tumors. Laparoscopy.

\section{INTRODUCCIÓN}

Si bien la primera nefrectomía parcial por tumor fue realizada en 1887 y descripta por Czerny (1) en el año 1890, la alta mortalidad del procedimiento, limitó su posterior indicación. En 1950 Vermooten (2) sugirió que las lesiones renales pequeñas, periféricas y encapsuladas, podrían ser resecadas con un margen de parénquima a su alrededor. Pero el diagnóstico de un tumor renal pequeño en aquellos años era un hecho excepcional. Robson publica la técnica de la nefrectomía radical en el año 1963 (3) y los resultados de la misma en el año 1969. Los buenos resultados obtenidos con la estandarización de ésta técnica quirúrgica, llevan a una franca mejoría de la sobrevida de los pacientes operados y a la aceptación de la nefrectomía radical para tratar cualquier tipo de tumor renal.

Las excepciones a ésta regla fueron aquellos pacientes en los cuales la nefrectomía los llevaría a la diálisis crónica. Ejemplo de esto son los tumores bilaterales, tumores en monorrenos, o con riñón contralateral patológico. Tanto en Estados Unidos como en Europa los resultados obtenidos con las cirugías conservadoras en tumores en estas circunstancias fueron tan buenos, que junto con la detección de tumores cada vez más pequeños dada por el uso masivo de la ecografía y la tomografía computada, impulsaron a diversos autores a realizar una nefrectomía parcial en tumores pequeños, con el riñón contralateral sano.

Varios trabajos demostraron en el largo plazo la equivalencia en el control oncológico de éstos pacientes tratados electivamente con una cirugía conservadora renal respecto de aquellos tratados con una nefrectomía radical $(4,5,6)$. Muchas de las masas renales pequeñas con iguales características radiológicas son finalmente patología benigna $(7,8)$, 
que de ser tratadas con una nefrectomía, representarían un claro ejemplo de sobretratamiento. En la actualidad, la cirugía conservadora de parénquima renal no solamente se considera equivalente a los resultados de la nefrectomía radical en los tumores pequeños, sino que presenta ventajas en la calidad de vida y en la sobrevida global de los pacientes $(9,10)$. Técnicamente el "gold standard" es la realización de ésta cirugía a cielo abierto, aunque la reproducción de ésta técnica por vía laparoscópica en pacientes más seleccionados, está demostrando iguales resultados con la ventaja de ser menos invasiva (11).

\section{MATERIAL Y MÉTODO}

Se revisaron las historias clínicas con análisis posterior de los datos obtenidos de aquellos pacientes, a los cuales se diagnosticó una masa ocupante renal sospechosa de tumor renal por ecografía y tomografía computada y se los trató con cirugía conservadora renal, en el Hospital Británico de Buenos Aires y Hospital Alemán desde el año 1995 y el Hospital Universitario Austral desde el año 2000. En la mayoría de los casos fue utilizada la técnica de nefrectomía parcial a cielo abierto (NP) o su equivalente que llamamos enucleorresección. En pocos pacientes se realizó una enucleación simple y a partir del año 2001 en pacientes muy seleccionados se realizó la técnica de nefrectomía parcial laparoscópica (NPL). El seguimiento de los pacientes fue a intervalos regulares con exámenes de laboratorio, radiografías de tórax y ecografías y/o tomografías computadas.

Se tabularon todos los datos de interés, se analizaron los resultados y se compararon con otras series de referencia.

\section{Técnica quirúrgica}

Para la nefrectomía parcial la vía de abordaje más utilizada fue la lumbotomía entre las 11 y 12 costillas. La correcta individualización de las fibras del diafragma y su desinserción es fundamental para no lesionar la pleura. La fascia de Gerota se incide extensamente, tratando durante la disección de dejar la grasa perirrenal adherida al tumor o, en su defecto, enviándola por separado para su examen histopatológico marcada en la superficie de contacto con el tumor. La liberación del riñón debe ser completa, lo que permite tenerlo casi a nivel de la piel. El examen visual y tactil se realiza en toda su superficie, en búsqueda de multicentricidad tumoral. Si se detecta algún nódulo pequeño, será el primero que se envíe a patología. Si bien el clampeo del pedículo no se realizó rutinariamente, en todos los casos se reparó el pedículo para poder clampearlo con facilidad en caso de necesidad. En todos estos pacientes se administró manitol aproximadamente 10 minutos previos al clampeo. Para la técnica de hibernación, hemos aislado con un campo plástico al riñón y aplicado hielo picado en toda la superficie renal, previo clampeo de la arteria renal o del pedículo en su totalidad. Esperamos generalmente 10 minutos de enfriamiento para luego proceder con una técnica de resección parcial clásica o enucleorresección, que consiste en marcar por fuera de los límites visibles de la lesión a unos $5 \mathrm{~mm}$ de la misma y considerando la forma a dar para facilitar la reconstrucción. Con instrumentos romos y tijeras se reseca la lesión rodeada de su pseudocápsula y un rodete de tejido renal sano a su alrededor. Examinamos en el momento la pieza operatoria en conjunto con el patólogo y realizamos una retoma del lecho solamente si hay sospechas en la pieza de alguna zona específica en que el tumor no presente margen de seguridad. El cierre de la vía excretora y la hemostasia de los vasos segmentarios la realizamos con una sutura continua de vicryl 4-0 que involucra ambas estructuras. En algunos casos se utilizó azul de metileno para individualizar la vía excretora a ser reparada.

En casos complejos donde se prevé una apertura amplia de la vía excretora o alguna dificultad en su reconstrucción insertamos previo a la cirugía un catéter doble jota para asegurar el drenaje de la orina y prevenir la formación de una fístula. La hemostasia global se logró con los puntos descriptos por Volker Moll (12), con vicryl 0. Aproximamos los cabos que surgen de éstos puntos interponiendo generalmente surgicel que se acomoda al lecho cruento. Revisamos la hemostasia por minutos luego de reperfundido el riñón. Rutinariamente dejamos un drenaje tubular ofrecido al lecho quirúrgico.

La vía laparoscópica la realizamos por vía transperitoneal, manoasistida o pura. Luego del decolamiento colónico, se realiza una prolija disección del pedículo reparando la arteria y vena renales con bandas elásticas. Disecamos luego el riñón, delimitando perfectamente el tumor y colocando algún trocar accesorio si fuera necesario para tener un buen ángulo de sutura para la reconstrucción del lecho. Clampeamos el pedículo, realizamos la exéresis tumoral y reconstrucción con sutura contínua y desclampeo precoz tal cual fuera descripto por Gill. Observamos en los minutos siguientes a la reperfusión renal la hemostasia y completamos la reconstrucción con algún agente hemostático. Dejamos un drenaje tubular en el lecho quirúrgico. 
TABLA I. CAUSAS DE NEFRECTOMÍA EN PACIENTES MONORRENOS.

\begin{tabular}{|lc|}
\hline Carcinoma renal & 15 \\
\hline Oncocitoma & 1 \\
\hline Tumor de Wilms & 1 \\
\hline Total & 17 \\
\hline
\end{tabular}

\section{RESULTADOS}

Se realizaron 254 cirugías conservadoras de riñón en un total de 246 pacientes de los cuales 73 fueron de sexo femenino y 173 fueron de sexo masculino, con un promedio de edad de 58 años. La vía convencional a cielo abierto (NP) fue utilizada en 220 procedimientos y la laparoscópica (NPL) a partir del año 2001 en 34. La indicación fue electiva o relativa en 236 procedimientos, ya que hubo 8 pacientes con tumores bilaterales y 18 pacientes monorrenos, de los cuales 15 fueron nefrectomizados previamente por adenocarcinoma renal, 1 paciente por tumor de Wilms), 1 paciente por un oncocitoma renal y otro paciente por anulacion funcional renal (Tabla I). El promedio del tamaño tumoral tratado fue de $3,49 \mathrm{~cm}$. El clampeo del pedículo se realizó en 168 pacientes, con hibernación en 43 pacientes con un promedio de 24,7 minutos.

El examen histopatológico demostró un carcinoma renal en 193 procedimientos. La mayoría de ellos correspondió al carcinoma convencional

\section{TABLA III. HISTOLOGÍA DE LAS LESIONES BENIGNAS $(n=67)$.}

\begin{tabular}{|ll|}
\hline Oncocitoma & 23 \\
\hline Angiomiolipoma & 17 \\
\hline Adenoma metanéfrico & 4 \\
\hline Quiste & 9 \\
\hline Adenoma papilar & 4 \\
\hline Leiomioma & 1 \\
\hline Pielonefritis crónica & 3 \\
\hline
\end{tabular}

\section{TABLA II. HISTOPATOLOGÍA DE LOS CARCINOMAS RENALES ( $n=193)$.}

\begin{tabular}{|lc|}
\hline Carcinoma convencional & 181 \\
\hline Carcinoma papilar & 9 \\
\hline Carcinoma cromófobo & 3 \\
\hline
\end{tabular}

( $n=181$ ), luego al carcinoma papilar $(n=9)$ y cromófobo ( $n=3$ ) (Tabla II) Las lesiones benignas se presentaron en 61 pacientes $(24 \%)$, la mayoría de ellas correspondieron a oncocitoma (23\%) luego según lo descripto en la Tabla III. Un paciente presentó márgenes quirúrgicos positivos en diferido, se le realizó la nefrectomía al mes y no se constató tumor residual. Un paciente presentó una imagen pseudotumoral del lecho quirúrgico por tomografía axial computada y resonancia magnética nuclear, se realizó una nefrectomía y no hubo hallazgo de tumor residual. Se presentaron complicaciones postoperatorias en 20 pacientes $(7,9 \%)$ (Tabla IV).

Las complicaciones hemorrágicas fueron las más frecuentes ( $n=9)$ Dos pacientes fueron reintervenidos inmediatamente, uno por sangrado del lecho que se solucionó con puntos hemostáticos y otro por lesión por trocar de la arteria epigástrica. De los tres

TABLA IV. COMPLICACIONES $n=20(7,9 \%)$.

\begin{tabular}{|ll|}
\hline Sangrado-hematoma & 9 \\
\hline Pseudoaneurisma & 2 \\
\hline Fístula urinaria & 3 \\
\hline Insuficiencia renal crónica & 1 \\
\hline Infección de herida & 1 \\
\hline Neumonía & 1 \\
\hline Lesión de uréter & 1 \\
\hline Urinoma & 1 \\
\hline Insuficiencia renal aguda & 1 \\
\hline
\end{tabular}


TABLA V. CAUSAS DE FALLECIMIENTO.

\begin{tabular}{|ll|}
\hline Progresión & 6 \\
\hline Cáncer de pulmón & 1 \\
\hline Cardiovasculares & 4 \\
\hline Sepsis & 1 \\
\hline
\end{tabular}

hematomas perinéfricos dos fueron observados y uno intervenido, mientras que un paciente monorreno luego de ser reintervenido tres veces por sangrado del lecho quedó en insuficiencia renal crónica por atrofia renal. Hubo dos sangrados tardíos al 5 y 20 días postoperatorios que fueron nefrectomizados. Dos pacientes presentaron hematuria y pseudoaneurismas. Uno fue tratado exitosamente con una embolización, el otro pese a la embolización y repetición del procedimiento presentó un nuevo sangrado y fue nefrectomizado. Un paciente monorreno con un fumor central, pese a una colocación prequirúrgica de catéter doble jota presentó una fístula por 20 días, infección de herida y una insuficiencia renal que no requirió diálisis. El tratamiento fue conservador, presentando el paciente luego de la recuperación post necrosis tubular aguda, una creatinina de 1,6 mg\%.

Otros dos pacientes presentaron filtración de orina por el drenaje más allá de las 72 hs con resolución espontánea de la misma. El control oncológico en el $84 \%$ de los pacientes con un promedio de 46,6 meses demostró una progresión con metástasis

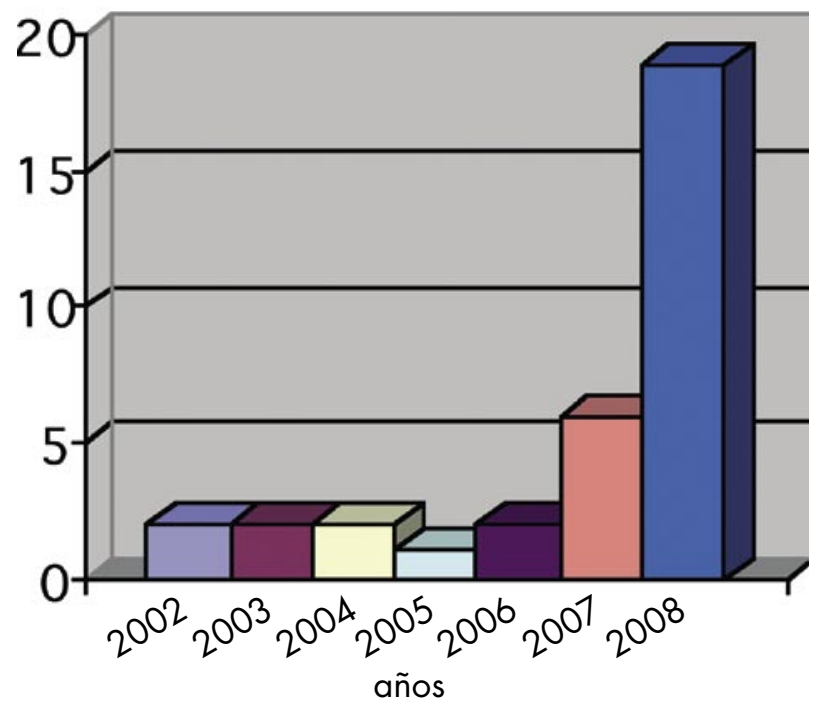

FIGURA 1. Indicaciones de laparoscopía por año.
TABLA VI. COMPARACIÓN DE LA NEFRECTOMÍA PARCIAL ABIERTA Y LAPAROSCÓPICA.

\begin{tabular}{|lcc|}
\hline & Abierta & Laparoscópica \\
\hline Edad & 58 años & 57 años \\
\hline Tamaño tumoral & $3,49 \mathrm{~cm}$ & $2,70 \mathrm{~cm}$ \\
\hline Tiempo de isquemia & $24,7 \mathrm{~min}$ & $30 \mathrm{~min}$ \\
\hline Lesiones benignas & $24 \%$ & $26 \%$ \\
\hline $\begin{array}{l}\text { Complicaciones } \\
\text { hemorrágicas }\end{array}$ & $2,4 \%$ & $8,8 \%$ \\
\hline
\end{tabular}

en 8 pacientes. La recurrencia local se observó en 4 pacientes, de los cuales en uno se realizó una nueva nefrectomía parcial, en otro una nefrectomía radical $y$ otros dos no fueron tratados, uno por mal estado general y progresión, el otro paciente se negó al tratamiento. Un paciente desarrolló un tumor en el riñón contralateral a los 20 meses y otro a los 10 años. La sobrevida específica por cáncer fue del $98 \%$ y la sobrevida global del $95 \%$. Los pacientes fallecidos fueron 6 por progresión de la enfermedad y 1 por cáncer de pulmón, 1 por sepsis y 4 por causas cardiovasculares (Tabla V).

En el último año el abordaje laparoscópico duplicó las indicaciones de todos los años previos (Figura 1). La edad de los pacientes fue comparable, el promedio del tamaño tumoral fue algo menor en las cirugías laparoscópicas $(3,49$ vs. $2,70 \mathrm{~cm})$ y el tiempo de clampeo algo más prolongado (30 vs. 24,7 min.). El porcentaje de lesiones benignas resecadas fue similar en ambos grupos de pacientes. Las complicaciones hemorrágicas fueron significativamente mayores en el grupo laparoscópico $(2,4 \%$ vs. $8,8 \%)$ (Tabla VI).

\section{DISCUSIÓN}

La indicación imperativa fue luego de la era pre-Robson aquella que nos mostró las primeras ventajas de la cirugía conservadora de parénquima renal en el tratamiento de tumores en relación a la nefrectomía y diálisis crónica. Ante ésta situación, el buen control oncológico, la calidad de vida y la sobrevida global del paciente en relación a un paciente anúrico en diálisis fue categórico (13).

La progresiva documentación de ésta situación (14) llevó a diversos autores a proponer la 
nefrectomía parcial en forma electiva (con el riñón contralateral sano) en pacientes con tumores renales pequeños. La detección de masas renales pequeñas, muchas veces incidentales, fue a partir de los años 80 cada vez más frecuente, y en muchos lugares son la forma más frecuente de presentación de los tumores de riñón (15). Seguimientos a 10 años han demostrado que los resultados oncológicos de la nefrectomía parcial son equivalentes a la nefrectomía radical (4). Muchas de las masas renales pequeñas detectadas actualmente son tumores benignos indiferenciables radiológicamente de un cáncer renal pequeño. En nuestra serie correspondió al $24 \%$ de los tumores operados, pero en otras series llegan al 30, 32 y $34 \%$ de las nefrectomías parciales realizadas. En todos estos casos una nefrectomía sacrificaría innecesariamente un riñón, dándose ésta situación en 2 a 3 de cada 10 pacientes operados. La biopsia renal percutánea tiene una especificidad del $90 \%$ para distinguir tumores renales benignos de malignos, del 80 a $90 \%$ para distinguir el subtipo patológico y del 50 a $75 \%$ para determinar el grado nuclear (17). 10 a $40 \%$ de los tumores renales sólidos menores de 4 $\mathrm{cm}$. biopsiados revelan ser benignos $(18,19)$. Hasta un $60 \%$ de éstos son oncocitomas y podrán ser también angiomiolipomas atípicos con escasa cantidad de tejido graso (20).

La diferenciación entre un oncocitoma y un carcinoma renal cromófobo puede ser dificultosa en la biopsia. Aparte de compartir algunas anomalías genéticas (pérdida del cromosoma 1 e Y) el carcinoma renal cromófobo puede estar asociado a un oncocitoma hasta en un 10\% de los casos. El 10\% de falla de la biopsia renal en diagnosticar un tumor maligno, lo hace un método poco seguro para solamente por el resultado de la biopsia no ofrecer al paciente un tratamiento curativo, máxime cuando el "gold standard" es una cirugía de exéresis tumoral con preservación del órgano, con la cual el diagnóstico del patólogo es $100 \%$ confiable y el tratamiento efectivo tanto para una patología benigna como maligna. Cuando la masa renal es indeterminada, una biopsia negativa no excluye la presencia de un carcinoma, por lo tanto no nos da la seguridad suficiente como para privar a un paciente de un tratamiento curativo.

Pero actualmente, aparte de la ventaja de la nefrectomía parcial de no sobretratar nefrectomizando innecesariamente a pacientes portadores de una masa renal pequeña, la ventaja teórica de conservar parénquima renal se plasma en la realidad, en una ventaja en la conservación de la función renal al largo plazo, con lo cual la posibilidad de desarrollar una insuficiencia renal es un 50\% menor en relación a los pacientes nefrectomizados $(21,22)$. A su vez hay estudios que demuestran que ésta situación lleva a una mejoría de la sobrevida de éstos pacientes respecto de los nefrectomizados, dados por una disminución de eventos cardiovasculares (910).

Un paciente de nuestra serie presentó márgenes quirúrgicos positivos en diferido y se le realizó la nefrectomía al mes y no se constató tumor residual. El impacto de un margen positivo sobre la posibilidad de recidiva ha sido estudiado en el Memorial Sloan Kettering Cancer Center (23). De 777 pacientes operados entre 1989 y 2005, 75 pacientes $(7,5 \%)$ tuvieron márgenes quirúrgicos positivos $(\mathrm{MQ}+)$, de los cuales $2(4 \%)$ desarrollaron recurrencia local. Mientras que de los 713 pacientes con MQ- negativos, $4(0,5 \%)$ desarrollaron una recidiva tumoral $(p=0.003)$. El estudio de Piper concluye que en un seguimiento de 60 meses el $28 \%$ de los $M Q+$ desarrollan una recidiva tumoral (24). Podemos concluir que un $M Q+$ da un riesgo aumentado de recidiva, pero que la mayoría de los pacientes seguirá libre de enfermedad, por lo que un seguimiento más estricto está indicado y no la nefrectomía. Probablemente algunos $M Q+$ sean un artefacto de técnica. La certificación de margen positivo por congelación ha sido duramente criticada.

Duvdevani (25) estudió 301 pacientes a los que realiza una nefrectomía parcial con examen por congelación de rutina del margen quirúrgico. 2 pacientes $(0,7 \%)$ tuvieron $M Q+$, y se realizó una nefrectomía en el momento. El examen diferido de las nefrectomías no detectó tumor residual. 4 pacientes tuvieron $M Q+$ en forma diferida y fueron observados. Durante un seguimiento de 1-10 años solamente 1 paciente tuvo una recidiva clínica, por lo que concluyen que el examen de $M Q$ por congelación carece de significado.

Otros dos estudios apoyan ésta afirmación $(26,27)$. Probablemente lo más importante sea la revisión macroscópica de la pieza operatoria, y una eventual biopsia de alguna zona sospechosa de $M Q_{+}$si existiere. La biopsia sistemática del lecho según Puppo es más una costumbre que una práctica con demostrado beneficio (28). La NPL la hemos realizado en 34 casos muy seleccionados de tumores periféricos, donde hemos planificado una excéresis sin clampeo del pedículo o con clampeo del mismo por menos de 30 minutos. La nefrectomía parcial laparoscópica (NPL) intenta duplicar la técnica a cielo abierto. Los principales problemas de la NPL son generalmente un mayor tiempo de clampeo y la falta de ayuda del tacto para delimitar la exéresis. La ecografía intraoperatoria puede ayudar a delimitar la lesión, pero el transductor para uso laparoscópico 
no está disponible en forma habitual. Las técnicas de hibernación si bien están descriptas (29), son de dificultosa implementación. La reconstrucción renal requiere un entrenamiento laparoscópico no solamente en técnicas ablativas sino también reconstructivas. Actualmente es conocido el peor pronóstico de un tumor mayor de $4 \mathrm{~cm}$, pero el tipo de nefrectomía (parcial vs. radical) no tendría impacto sobre la sobrevida específica por cáncer en pacientes con Tl mayores de $4 \mathrm{~cm}$, por lo cual algunos autores proponen extender las indicaciones de ésta técnica para todos los $\mathrm{Tl}$ pasibles de realizar una nefrectomía parcial (30).

\section{CONCLUSIONES}

La cirugía conservadora renal, actualmente considerada el gold standard del tratamiento de los tumores renales pequeños, es una técnica segura y confiable para el tratamiento de dichos tumores. Las ventajas de la utilización de ésta técnica sobre la nefrectomía radical se han ido fortaleciendo. La evolución tecnológica nos permite para casos seleccionados reproducir la cirugía a cielo abierto por la vía laparoscópica, obteniendo similares resultados.

\section{BIBLIOGRAFIAA y LECTURAS RECOMENDADAS ( ${ }^{*}$ lectura de interés $y^{* *}$ lectura fundamental)}

1. Czerny H E. Citado por Herczel E. Ueber Nierenextirpation Beitr. 2. Klin Chir, 1890; 6: 485

2. Vermooten V. Indications for conservative surgery in certain renal tumors: a study based on the growth pattern of the clear cell carcinoma. J Urol, 195064: 200.

3. Robson C J. Radical nephrectomy for renal cell carcinoma. J Urol, 1963 89: 37-42.

**4. Harry W Herr. Partial nephrectomy for unilateral renal carcinoma and a normal contralateral kidney: 10-year follow-up. J Urol. 1999; 161:33.

**5. Weber KO Lau, Michael L Blute, Amy L Weaver, Vicente E Torres, Horst Zincke. Matched comparision of radical nephrectomy versus nephron sparing surgery in patients with unilateral renal cell carcinoma and a normal contralateral kidney. Mayo Clin Proc. 2000; 75: 1236-42.

*6. Cherryl T Lee, Jared Katz, Weiji Shi, Howard T. Thaler, Victor E. Reuter, Paul Russo: Surgical management of renal tumors $4 \mathrm{~cm}$.or less in a contemporary cohort. J Urol, 2000; 163, 730-36.

7. Van Poppel, Am Urol Assoc Update Series, 1994; 31:246.

8. Novick AC, Campbell SC. Renal tumors. Campbell's Urology. Philadelphia: Saunders; 2002, pp.2672-731
**9. Houston Thompson, Stephen A Boorjian, Christine M Lohse, Bradley C Leibovich, Eugene D Kwon, John C Cheville, Michael Blute. Radical Nephrectomy for pT1a Renal Masses may be associated with decreased overall survival compared with partial nephrectomy. J Urol, 2008; 179, 468.

10. William C Huang, Elena B Elkin, Thomas L Jang, Paul Russo. Radical nephrectomy is associated with increased mortality in patients with small renal tumors. Abstract \# 493, J Urol 2007; 177, 164.

**11. Inderbir S Gill, Louis R Kavoussi, Brian R Lane, Michael L Blute, Denise Babineau, J Roberto Colombo et al. Comparison of 1800 laparoscopic and open partial nephrectomies for single renal tumors. J Urol, 2007; 178 (1) 41-6.

12. Moll V, Becht E and Ziegler M. Kidney preserving surgery in renal cell tumors: indicattions, thechniques and results in 152 patients. J Urol, 1993; 150:319-23

13. Novick A, Streem S, Montie J, Pontes JE, Siegel $\mathrm{S}$, Montague DK et al. Conservative surgery for renal cell carcinoma: a single center experience with 100 patients. J Urol, 1989; 141:835-9.

14. Pérez García F J, Regadera Sejas F J, Rodríguez Martínez J J, Martínez Gómez F J, Casasola Chamorro J, Sánchez Trilla A. Adenocarcinoma renal bilateral sincrónico. Nuestra experiencia con la cirugía conservadora renal. Arch Esp de Urol, 1999; 52(5):471-7.

**15. Allan J. Pantuck, Amnon Zisman, Arie S Belldegrun. The changing natural history of renal cell carcinoma. J Urol, 2001; 166, 1611-23.

16. Mayo Clinic, Cleveland Clinic, Johns Hopkins, Pantuck AUA 2007.

17. Eric Lechevallier. Core biopsy of solid renal masses under CT guidance. European Urology Supplements 6, 2007; 540-3.

18. Vasudevan A, Davies RJ, Shannon BA, Cohen RJ. Incidental renal tumors: the frequency of benign lesions and the role of preoperative core biopsy. BJU Int 2006; 97:946-9.

19. Neuzillet Y, Lechevallier E, Andre M, Daniel L, Coulange C. Accuracy and clinical role of fine needle percutaneous biopsy with computerized tomography guidance of small (less than $4 \mathrm{~cm}$ ) renal masses. J Urol 2004;171:1802-5.

20. DA Barocas, SM Rohan, J Kao, RD Gurevich, JJ del Pizzo, ED Vaughan, et al. Diagnosis of renal tumors on needle biopsy specimens by histological and molecular analyzis. J Urol, 2006; 176:1957-62.

21. Dash A, Vickers AJ, Schachter LR, Bach AM, Snyder ME, Russo P. Comparison of outcomes in elective partial vs radical nephrectomy for clear cell renal cell carcinoma of 4-7 cm. BJU Int 2006; 97(5) 939-45 . 
*22. McKiernan J, Simmons R, Katz J, Russo P. Natural history of chronic renal insufficiency after partial and radical nephrectomy. Urology, 2002; 59(6):816-20.

23. Kwon EO, Carver BS, Snyder ME, Russo P. Impact of positive surgical margins in patients undergoing partial nephrectomy for renal cortical tumours. BJU Int 2007 Feb;99(2):286-9. Epub 2006 Nov 28. Citado Eur Urol Suppl 6, 2007, 539.

24. Piper NY, Bishoff JT, Magee C, Haffron JM, Flanigan $\mathrm{RC}$, Mintiens $\mathrm{A}$, et al. Is a $1 \mathrm{~cm}$ margin necessary during nephron-sparing surgery for renal cell carcinoma? Urology, 2001; 58(6): 849-52.

25. Mordechai Duvdevani, Menachem Laufer, Alexander Kastin, Yoram Mor, Andrei Nadu, Jacob Hanani et al. Is frozen section analysis in nephron sparing surgery necessary? A clinicopathological study of 301 cases. J Urol, 2005; 173:385-7.

26. Kubinski DJ, Clark PE, Assimos DJ, Hall MC. Utility of frozen section analysis of resection margins during partial nephrectomy. Urology, 2004; 64(1):31-4.
27. Timsit MO, Bazin JP, Thiounn N, Fontaine E, Chretien Y, Dufour B, et al. Prospective study of safety margins in partial nephrectomy: intraoperative assessment and contribution of frozen section analysis. Urology 2006 May;67:923-6.Epub 2006 Apr 25.

28. Puppo P, Introini C, Calvi P, Naselli A. Long term results of excision of small renal cancer surrounded by a minimal layer of grossly normal parenchima; review of 94 cases. Eur Urol 2004; 46(4):477-81.

29. Inderbir S Gill, Sidney C Abreu, Mihir M Desai, Andrew P Steimberg, Anup P Ramani, Christopher NG et al. Laparoscopic ice slush renal hipothermia for partial nephrectomy: the initial experience. J Urol, 2003; 170(1):52-6.

**30. Patard JJ, Shvarts O, Lam JS, Pantuck A, Kim HL, Ficarra V, et al. Safety and efficacy of partial nephrectomy for all T1 tumors based on an international multicenter experience. J Urol, 2004; 171, 2181-5. 